\title{
Percepção de sono: duração, qualidade e alerta em profissionais da área de enfermagem
}

\author{
How nursing staff perceive the duration \\ and quality of sleep and levels of alertness
}

Frida Marina Fischer 1

Liliane Reis Teixeira 1

Flavio Notarnicola da Silva Borges 1

Mariana Brandão Lourenço Gonçalves 1

Regiane Miranda Ferreira 1

1 Departamento de Saúde Ambiental, Faculdade de Saúde Pública, Universidade de São Paulo. Av. Dr. Arnaldo 715, São Paulo, SP 01246-904, Brasil. fmfische@usp.br

\begin{abstract}
This study was conducted among health care personnel (registered nurses and nurse aides) in a public hospital in São Paulo, Brazil. Work was organized in 12-hour daytime or nighttime shifts, followed by 36 hours off. The study aimed to evaluate how the nursing staff perceived the duration and quality of sleep both during and off work days, as well as their perception of alertness during working hours. There were significant differences between night and day in the duration of sleep (Student $t$ test $=10.82 ; p<0.000$ ). Quality of daytime sleep after working night shifts was perceived as worse than nighttime sleep (Wilcoxon test, $Z=2.67 ; p<0.007$ ). Significant differences were detected in self-evaluation of alertness after the $2 \mathrm{nd}, 6 \mathrm{th}$, and 10th hour of night shifts (Friedman $=63.0 ; p<0.00$ ). Alertness was perceived as worse during dawn hours. This is an indication of sleepiness at work and can have serious consequences for both health care workers and patients.
\end{abstract}

Key words Shift Work; Nurses; Nurses' Aides; Sleep; Arousal

Resumo Foi realizado um estudo entre auxiliares de enfermagem e enfermeiros que trabalhavam em hospital público de São Paulo. A organização dos turnos diurnos e noturnos fixos era de 12 horas diárias, seguidas de 36 horas de descanso. O objetivo deste estudo foi avaliar a percepção da duração e qualidade dos episódios de sono nos dias de trabalho e de descanso, bem como dos níveis de alerta durante os turnos diurnos e noturnos de 12 horas de trabalho. Comparadas as durações dos episódios de sono, foram detectadas diferenças significativas entre sono diurno $e$ noturno (Teste t de Student $=10,82 ; p<0,000$ ). A qualidade dos episódios de sono diurno após as noites de trabalho foi percebida como pior do que a qualidade dos episódios de sono noturno (Teste de Wilcoxon, $Z=2,67 ; p<0,007$ ). Foram encontradas diferenças significativas na percepção dos estados de alerta em três momentos diferentes do turno da noite (Friedman = 63,0; $p<$ 0,00). Os níveis percebidos de alerta à noite tornam-se piores à medida que aumenta o número de horas de trabalho. Isso é um indicativo de que a sonolência no trabalho noturno se faz presente e pode prejudicar seriamente tanto trabalhadores quanto os pacientes que estão aos seus cuidados.

Palavras-chave Trabalho em Turnos; Enfermeiros; Auxiliares de Enfermagem; Sono; Nível de Alerta 


\section{Introdução}

Entre os mais antigos grupos profissionais que trabalham em sistemas de turnos, encontramse os dos serviços de saúde, dentre os quais os enfermeiros e os auxiliares de enfermagem.

As escalas de trabalho em hospitais são geralmente organizadas em turnos fixos contínuos, uma vez que os serviços dessas instituições exigem um funcionamento ininterrupto durante as $24 \mathrm{~h}$ do dia, sete dias por semana. No Brasil, já é uma tradição adotar-se, para o corpo de enfermagem, o turno de $12 \mathrm{~h}$ de trabalho diário (diurno ou noturno), seguido de 36 horas de descanso.

Vários autores estudaram as conseqüências do trabalho em turnos no ciclo vigília-sono dos trabalhadores da área da saúde. Pesquisa realizada com 970 enfermeiros selecionados aleatoriamente em hospitais públicos de Valência, Espanha, mostrou que, para ambos os sexos, o trabalho em turnos conduz a uma redução na duração de sono e alteração na qualidade deste (Escribà et al., 1992).

Estryn-Behar et al. (1978) realizaram estudos envolvendo profissionais de enfermagem do sexo feminino e constataram que, entre as do turno da noite, houve uma redução da duração de sono diurno nos dias de trabalho, com uma média de horas referida inferior àquela registrada pelas mulheres trabalhadoras no turno diurno. A realização de atividades domésticas levava à fragmentação do sono diurno e atraso na hora de dormir após o plantão noturno, implicando uma menor duração total do sono, mesmo nos dias de folga, nos quais o período de sono à noite era mais extenso.

A restrição de atividades de lazer foi referida pelas trabalhadoras noturnas, que, surpreendentemente, informaram não ter tempo de cuidar dos filhos, embora esta tenha sido a razão apontada para a escolha deste turno de trabalho (Menezes, 1996).

Estudos mostram que os efeitos acumulados durante uma semana, com uma média de duração de sono de $4,5 \mathrm{~h}$ por dia, levam a sonolência a níveis similares ao da privação total de sono, aumentando consideravelmente as chances de ocorrerem acidentes de trabalho (Dinges et al., 1997). Em estudo feito com 678 enfermeiras de um hospital norte-americano, Gold (1992) relatou haver um risco de acidentes relacionados a cochilos ou à sonolência durante o plantão ou ao volante, na ida ao trabalho ou no retorno deste.

O estudo do ciclo vigília-sono pode ainda detectar um ajuste do sistema circadiano ao longo do tempo de trabalho noturno. Sabendo- se que esse ajuste é apenas parcial, pode-se determinar a gravidade e a intensidade das principais alterações do sono e dos sintomas provocados pelo trabalho noturno. Niedhammer et al. (1994) estudaram uma população de 469 profissionais de enfermagem de seis hospitais públicos da França. Foram avaliadas, numa população feminina, durante dez anos, as relações entre turno de trabalho e qualidade de sono. Os resultados mostraram que profissionais de enfermagem têm mais distúrbios de sono em escalas alternadas quando comparados com os de escalas fixas. Um outro resultado apontou uma queda na incidência dos distúrbios de sono após a transferência de algumas enfermeiras para o turno diurno.

No Brasil, poucos estudos foram realizados nesse campo. Destaca-se o feito por Menezes (1996), que estudou enfermeiras de um hospital público de Salvador, Bahia. O estudo mostrou que, com base na auto-avaliação de saúde das profissionais, quando as enfermeiras noturnas foram comparadas às dos turnos diurnos, com ou sem experiência prévia de turno da noite, as primeiras apresentaram taxas mais elevadas de prevalência de sintomas e problemas de saúde que as diurnas. Os sintomas estavam associados ao trabalho noturno, tais como: alteração da qualidade de sono, distúrbios digestivos e sintomas de fadiga.

\section{Objetivos}

Analisar a percepção da duração e da qualidade dos episódios de sono nos dias de trabalho e de descanso, bem como os níveis de alerta em dias de trabalho, entre auxiliares de enfermagem e enfermeiros, que trabalhavam em turnos fixos de 12 horas, diurnos e noturnos.

\section{Metodologia}

\section{População de estudo}

Um estudo abrangente sobre condições de vida e trabalho e repercussões no envelhecimento funcional precoce (também chamado de "envelhecimento e trabalho") foi conduzido no período de 1997 a 1999, no Instituto Central do Hospital das Clínicas (ICHC) da Faculdade de Medicina da Universidade de São Paulo (FMUSP) (Fischer et al., 2002). Esse Instituto possui 1.684 trabalhadores da área da saúde (350 enfermeiros, 1.178 auxiliares de enfermagem e 156 atendentes de enfermagem) que trabalham em sistema de turnos. Somente os 255 funcionários 
dos setores de Emergências Clínicas (enfermaria, UTI e pronto-socorro) foram convidados a participar deste estudo. Esses setores atendem 450 pacientes por dia.

Dos 255 trabalhadores convidados, 176 pessoas (69\% da população) aceitaram participar da pesquisa mencionada acima, respondendo inicialmente aos questionários: Índice de Capacidade para o Trabalho (Tuomi et al., 1997) e Envelhecimento e Trabalho (Tepas, 1996).

A segunda etapa da pesquisa "envelhecimento e trabalho" iniciou-se um ano após a primeira e teve como principal objetivo avaliar a percepção do sono dos trabalhadores em turnos que participaram da primeira etapa. Quarenta e cinco pessoas aceitaram participar do estudo do sono, entretanto este trabalho relata os resultados de somente 26 pessoas que trabalhavam em turnos de $12 \mathrm{~h}$ (diurno ou noturno) seguidas por 36 horas de descanso. As demais pessoas $(n=19)$ trabalhavam em outros esquemas de turnos: 13 em turnos de seis ou oito horas diurnas, e seis mencionaram ter mais de um emprego em turnos diurnos de seis ou oito horas no ICHC/FMUSP, combinados com turnos de $12 \mathrm{~h}$ em outra instituição (apenas uma trabalhava em turnos de $12 \mathrm{~h}$ noturnas). Por serem os turnos de $12 \mathrm{~h}$ bastante usuais entre os trabalhadores da área de enfermagem no Brasil, e devido a controvérsias existentes na literatura sobre a adoção de jornadas extensas de trabalho em turnos e as possíveis conseqüências à saúde, decidiu-se prioritariamente publicar os resultados deste grupo.

\section{Princípios éticos}

Foram incluídos na amostra todos aqueles trabalhadores que, após abordagem pessoal, aceitaram participar voluntariamente do estudo. Os dados individuais foram mantidos sob sigilo, somente disponíveis para o próprio participante. Essa aceitação foi documentada, por meio do preenchimento de um termo de consentimento esclarecido que foi assinado pelo trabalhador. A pesquisa foi aprovada pelo Comitê de Ética da Faculdade de Saúde Pública/Universidade de São Paulo em 15 de maio de 1998.

\section{Instrumentos de coleta de dados}

- Protocolos diários de atividade e escalas visuais analógicas: Os protocolos são divididos em unidades de tempo de 15 minutos cada, e em cinco atividades: (1) sono; (2) outras atividades - preenchidas somente por pessoas que trabalhavam em um segundo emprego fora do
Hospital das Clínicas ou que estudavam; (3) folga - atividades não relacionadas ao trabalho; (4) transporte; (5) trabalho. Os indivíduos foram instruídos a preencher os protocolos, durante 15 dias consecutivos, fazendo um traço contínuo no mesmo, de acordo com as atividades diárias e tempo gasto em cada uma delas. Esse protocolo foi originalmente idealizado por Knauth et al. (1983) e adaptado e utilizado em outros estudos por Fischer et al. (1987). Cada protocolo apresenta também quatro escalas analógicas visuais, de $10 \mathrm{~cm}$ cada, precedidas pela pergunta “Como você dormiu?" A escala é iniciada em "muito mal” (zero) e finalizada em "muito bem" (dez). Para cada episódio de sono, os indivíduos assinalaram com um traço vertical, registrando suas percepções quanto à qualidade do sono. Nos dias de trabalho, foram anexadas aos protocolos três páginas com uma escala analógica visual de $10 \mathrm{~cm}$ em cada uma delas. Cada escala foi precedida pela pergunta: " $O$ quão alerta você se sente nesse momento?”. Essas escalas foram usadas para avaliar a percepção do nível do alerta do trabalhador em três momentos do turno (segunda, sexta e décima hora de trabalho). Para o turno da noite, os registros ocorreram às $21 \mathrm{~h}, 1 \mathrm{~h}$ e $5 \mathrm{~h}$, e no período diurno às $9 \mathrm{~h}, 13 \mathrm{~h}$ e $17 \mathrm{~h}$. A escala é iniciada pela expressão "muito sonolento" (zero) e finalizada por "muito alerta" (dez); os indivíduos assinalaram a escala com um traço vertical de acordo com suas percepções dos estados de alerta. A avaliação da percepção do alerta foi avaliada da mesma forma que a da qualidade do sono.

- Análise estatística: Os dados obtidos nos protocolos diários de atividade foram digitados em planilhas usando o programa Excel 7.0. Foram calculadas médias e desvios-padrão para as variáveis duração, qualidade e alerta, para cada turno. Para as análises estatísticas, "o dia" dos indivíduos que trabalhavam à noite foi estipulado das $19 \mathrm{~h}$ às $18 \mathrm{~h} 59 \mathrm{~min}$ do dia subseqüente; e "o dia" daqueles que trabalharam durante o turno diurno foi das $0 \mathrm{~h}$ às $23 \mathrm{~h} 59 \mathrm{~min}$.

Foram escolhidas para análise apenas duas categorias de sono em cada turno. Isso ocorreu devido ao fato de algumas das classes não possuírem um número de episódios de sono suficiente para análises estatísticas. Para avaliar o turno de $12 \mathrm{~h}$ de trabalho noturno, foram escolhidas as classes "Sono Noturno" (SN) e "Sono Diurno em Dias de Trabalho Noturno” (SDCTN). Para o turno de $12 \mathrm{~h}$ de trabalho diurno, as classes escolhidas foram: "Sono Noturno em Dias de Descanso" (SNSTD) e "Sono Noturno em Dias de Trabalho Diurno" (SNCTD). A normalidade dos resultados foi avaliada pelo Teste de Shapiro-Wilk, e a homogeneidade de variância, pelo 
Teste de Levene. Os dados de duração e qualidade de sono que mostraram homogeneidade de variância foram avaliados pelo Teste $t$ de Student para amostras dependentes no programa Statistica (Stat Soft Inc., 1995). Já os resultados que não obtiveram homogeneidade de variância foram testados pela prova não paramétrica de Wilcoxon.

Os dados do alerta que registraram homocedasticidade foram testados em uma análise de variância (ANOVA), ao passo que aqueles que não registraram homogeneidade de variância foram testados pela prova não paramétrica de Friedman (Fonseca \& Martins, 1996; Siegel, 1975).

\section{Resultados}

\section{Características individuais}

da população estudada

Os dados de todas as pessoas mencionadas neste artigo referem-se somente àquelas que trabalhavam em turnos 12h diárias seguidas de 36 horas de descanso, dos quais 11 estavam em turnos diurnos (7h-19h) e 15, em turnos da noite (19h-7h).

A idade média dos 26 trabalhadores - 24 mulheres $(92,3 \%)$ e dois homens $(7,7 \%)-$, cujos relatos de sono são discutidos neste artigo, era de 37,3 anos (desvio-padrão = 7,5 anos). Sua ocupação era de: auxiliares de enfermagem (20), atendentes de enfermagem (4) e enfermeiros de nível superior (2). Estado marital: cinco eram solteiros, 16 viviam com um(a) companheiro(a) ou eram casados, dois eram divorciados e três eram viúvos. Vinte e uma pessoas $(80,8 \%)$ relataram ter filhos; dentre as mulheres estudadas, sete tinham filhos menores que quatro anos (cinco com uma criança e duas com duas crianças). Onze pessoas tinham filhos entre 5 e 11 anos. Quanto à escolaridade: dez tinham apenas o primeiro grau completo; um tinha curso técnico de primeiro grau; dois tinham segundo grau incompleto; cinco, segundo grau completo; dois, curso técnico de segundo grau; quatro, curso superior incompleto; um, curso superior completo; havia um com pós-graduação (mestrado). Em relação a alguns hábitos de vida: $23,1 \%$ (6) eram fumantes; $30,8 \%$ (8) faziam exercício regularmente; $4(15,4 \%)$ relataram consumo freqüente de bebida alcoólica.

A qualidade da residência foi considerada adequada para o repouso por seis trabalhadores (três diurnos e três noturnos) e inadequada por dez outros (cinco diurnos e cinco noturnos); os demais relataram condições intermediárias (três diurnos e sete noturnos). Em rela- ção ao ruído na residência: apenas quatro relataram não ter esse problema (um diurno e três noturnos); 13 têm algum problema (cinco diurnos e oito noturnos) e nove mencionaram que o ruído é um incômodo importante atrapalhando o repouso (cinco diurnos e quatro noturnos). Em relação ao número de quartos para dormir: a maioria (24) tem seu próprio quarto de dormir.

\section{Duração do sono - $12 \mathrm{~h}$ de trabalho noturno seguidas de $36 \mathrm{~h}$ de descanso}

Realizado o Teste de Shapiro-Wilk, verificou-se haver normalidade na distribuição dos resultados referentes à duração do sono noturno $(\mathrm{p}=$ $0,05)$; o mesmo não foi observado para os dados da duração do sono diurno $(p=0,33)$. Foi encontrada homocedasticidade na duração dos episódios de sono dos indivíduos ( $\mathrm{F}=0,18$; $\mathrm{p}=0,67$ ). Comparando os episódios de sono noturno e sono diurno em dias de trabalho noturno, foram encontradas diferenças significantes entre ambos, de acordo com o Teste t de Student $(\mathrm{t}=10,82$ e $\mathrm{p}<0,00)$, ou seja, a média de duração do sono noturno é maior que a média de duração do sono diurno em dias de trabalho (Tabela 1).

\section{Duração do sono - $12 \mathrm{~h}$ de trabalho diurno seguidas de $36 \mathrm{~h}$ de descanso}

Para a duração do sono noturno em dias de trabalho diurno e nos dias de descanso dos indivíduos, foi realizado o Teste de Shapiro-Wilk. Não foi verificada normalidade $(p=0,51)$ na duração do sono noturno em dias de trabalho diurno; em dias de descanso foi encontrada normalidade $(p=0,00)$. Foi verificada homocedasticidade entre a duração dos episódios de sono dos indivíduos ( $\mathrm{F}=0,02 ; \mathrm{p}=0,87$ ). Comparando os episódios de sono noturno foram encontradas diferenças significantes (Teste $\mathrm{t}$ de Student, $\mathrm{t}=2,20, \mathrm{p}<0,05$ ) entre as duas classes analisadas, ou seja, a média de duração de sono noturno em dias de trabalho diurno é maior que a média de duração do sono noturno em dias de descanso (Tabela 1).

\section{Qualidade do sono - $12 \mathrm{~h}$ de trabalho noturno seguidas de $36 \mathrm{~h}$ de descanso}

Por meio do Teste Shapiro-Wilk para a qualidade dos episódios de sono, verificou-se normalidade na distribuição das médias $(\mathrm{p}=0,00)$; utilizando a análise de variância, não foi encontrada homocedasticidade entre os episódios de sono $(F=7,51 ; p=0,01)$. Para a compa- 
Média e desvio-padrão (em minutos) da duração do sono de indivíduos que trabalham em turnos:

12h de trabalho diurno ou noturno, seguidas de 36h de descanso. Hospital das Clínicas, São Paulo, 1999.

\begin{tabular}{|c|c|c|c|c|c|c|c|}
\hline \multirow[b]{3}{*}{$\begin{array}{l}\text { Episódio } \\
\text { de sono }\end{array}$} & \multicolumn{7}{|c|}{ Duração do sono } \\
\hline & \multicolumn{3}{|c|}{ Trabalho noturno } & \multirow[b]{2}{*}{$\begin{array}{l}\text { Episódio } \\
\text { de sono }\end{array}$} & \multicolumn{3}{|c|}{ Trabalho diurno } \\
\hline & $\mathrm{n}$ & Média & DP & & $\mathrm{n}$ & Média & DP \\
\hline SN & 105 & 471,21 & 176,48 & SD & 24 & 124,42 & 106,25 \\
\hline SDCTN & 78 & 208,73 & 92,57 & SNCTD & 79 & 458,92 & 125,45 \\
\hline SDSTN & 27 & 149,26 & 111,69 & SNSTD & 47 & 333,19 & 100,82 \\
\hline SNF & 32 & 497,09 & 108,98 & SNF & 15 & 443,80 & 91,09 \\
\hline SDF & 6 & 125,67 & 72,02 & SDF & 3 & 84,00 & 60,62 \\
\hline SNOT & 23 & 109,17 & 97,89 & - & - & - & - \\
\hline
\end{tabular}

$\mathrm{n}$ = número de episódios de sono; $\mathrm{SN}$ = sono noturno em dias de descanso (entre 19h e 6h59min);

SDCTN = sono diurno em dias de trabalho noturno (entre $7 \mathrm{~h}$ e $18 \mathrm{~h} 59 \mathrm{~min}$ ), quando houve trabalho noturno

na noite anterior; SDSTN = sono diurno em dias de descanso (entre $7 \mathrm{~h}$ e $18 \mathrm{~h} 59 \mathrm{~min}$ ), quando não houve trabalho noturno na noite anterior; SNF = sono noturno em dias de folga (entre 19h e 6h59min); SDF = sono diurno em dias de folga (entre $7 \mathrm{~h}$ e $18 \mathrm{~h} 59 \mathrm{~min}$ ); SNOT = sono no trabalho (entre $19 \mathrm{~h}$ e $7 \mathrm{~h}$ ); SD = sono diurno em dias de descanso (entre $7 \mathrm{~h}$ e $18 \mathrm{~h} 59 \mathrm{~min}$ ); SNCTD = sono noturno em dias de trabalho diurno (entre 19h e $6 \mathrm{~h} 59 \mathrm{~min}$ ), quando houve trabalho durante o dia; SNSTD = sono noturno em dias de descanso (entre $19 \mathrm{~h}$ e $6 \mathrm{~h} 59 \mathrm{~min}$ ). Os dias de folga foram considerados a partir de $36 \mathrm{~h}$ após o término do trabalho.

ração da qualidade do sono foi então utilizado o Teste de Wilcoxon, e foram detectadas diferenças significativas $(Z=2,67$ e $\mathrm{p}<0,00)$. Assim sendo, pode-se afirmar que a percepção da qualidade do sono noturno em dias de descanso é maior que a qualidade do sono diurno em dias de trabalho noturno (Tabela 2).

\section{Qualidade do sono - $12 \mathrm{~h}$ de trabalho diurno seguidas de $36 \mathrm{~h}$ de descanso}

Utilizando o Teste de Shapiro-Wilk, foi analisada a qualidade do sono noturno. Encontrou-se normalidade na distribuição das médias ( $\mathrm{p}=$ $0,00)$. Na análise de variância, foi verificada homocedasticidade entre a qualidade dos episódios de sono $(F=0,23 ; p=0,63)$. Utilizando o Teste $t$ de Student, não foram detectadas diferenças significativas $(t=1,84$ e $p=0,09)$ entre as medidas da qualidade percebida dos episódios de sono noturno. Assim sendo, a qualidade do sono noturno em dias de trabalho diurno é semelhante à qualidade do sono noturno em dias de descanso (Tabela 2).

Estado de Alerta Subjetivo - $12 \mathrm{~h}$ de trabalho noturno seguidas de $36 \mathrm{~h}$ de descanso

A auto-avaliação do alerta dos indivíduos que trabalham no turno da noite apresentou maior média às $21 \mathrm{~h}$ horas (média: 8,58 ; desvio-padrão: 1,57). Com o decorrer das horas de trabalho, ocorreu uma queda sensível, atingindo, às 5h, a média 5,04 e desvio-padrão 3,30 (Tabela
3). As variações do desvio-padrão entre os turnos diurno e noturno podem ser observadas na Figura 1. O desvio-padrão das respostas referentes ao alerta percebido durante o turno diurno apresentou poucas variações no decorrer das horas de trabalho. Por outro lado, durante o turno da noite o desvio-padrão do alerta percebido aumentou à medida que se passavam as horas de trabalho. O Teste Shapiro-Wilk para os estados de alerta relatados às $21 \mathrm{~h}, 1 \mathrm{~h}$ e $5 \mathrm{~h}$ indicou normalidade na distribuição $(\mathrm{p}=0,00)$. Na análise de variância, não foi verificada homogeneidade $(F=30,432 ; p=0,00)$. Uma vez que não ocorreu homogeneidade das variâncias analisadas pelo Teste de Levene, as medidas foram comparadas pela prova não paramétrica de Friedman. Há diferenças significativas entre as percepções de alerta registradas durante os três momentos $\left(\chi^{2}=63,00714 ; \mathrm{p}<0,00 ; \mathrm{N}=\right.$ $74 ; \mathrm{df}=2$ ).

\section{Estado de Alerta Subjetivo - 12h de trabalho diurno seguidas de $36 \mathrm{~h}$ de descanso}

A auto-avaliação do alerta durante o turno diurno apresentou médias muito próximas para os três horários (9h, 13h e 17h) (Tabela 3). O Teste de Shapiro-Wilk mostrou haver normalidade na distribuição dos estados de alerta $(p=0,00)$. $\mathrm{Na}$ análise de variância, foi verificada homocedasticidade para o estado de alerta nos três momentos analisados $(\mathrm{F}=0,64 ; \mathrm{p}=0,52)$. Os resultados foram comparados por intermédio da Análise de Variância (ANOVA), que indicou não 
Média e desvio-padrão da qualidade do sono de indivíduos que trabalham em turnos de $12 \mathrm{~h}$ de trabalho diurno ou noturno, seguidas de $36 \mathrm{~h}$ de descanso. Hospital das Clínicas, São Paulo, 1999.

\begin{tabular}{|c|c|c|c|c|c|c|c|}
\hline \multirow[b]{3}{*}{$\begin{array}{l}\text { Episódio } \\
\text { de sono }\end{array}$} & \multicolumn{7}{|c|}{ Qualidade do sono } \\
\hline & \multicolumn{3}{|c|}{ Trabalho noturno } & \multirow[b]{2}{*}{$\begin{array}{l}\text { Episódio } \\
\text { de sono }\end{array}$} & \multicolumn{3}{|c|}{ Trabalho diurno } \\
\hline & $n$ & Média & $\mathrm{DP}$ & & $\mathrm{n}$ & Média & DP \\
\hline SN & 92 & 7,76 & 2,37 & $\mathrm{SD}$ & 20 & 6,06 & 2,72 \\
\hline SDCTN & 71 & 5,23 & 3,44 & SNCTD & 74 & 7,22 & 2,65 \\
\hline SDSTN & 22 & 4,49 & 3,35 & SNSTD & 42 & 6,11 & 2,58 \\
\hline SNF & 27 & 7,89 & 1,80 & SNF & 15 & 6,07 & 2,06 \\
\hline SDF & 3 & 5,27 & 4,37 & SDF & 2 & 5,65 & 0,78 \\
\hline SNOT & 18 & 5,45 & 2,16 & - & - & - & - \\
\hline
\end{tabular}

$\mathrm{n}$ = número de episódios de sono; $\mathrm{SN}$ = sono noturno em dias de descanso (entre $19 \mathrm{~h}$ e $6 \mathrm{~h} 59 \mathrm{~min}$ );

SDCTN = sono diurno em dias de trabalho noturno (entre $7 \mathrm{~h}$ e $18 \mathrm{~h} 59 \mathrm{~min}$ ), quando houve trabalho noturno

na noite anterior; SDSTN = sono diurno em dias de descanso (entre $7 \mathrm{~h}$ e $18 \mathrm{~h} 59 \mathrm{~min}$ ), quando não houve trabalho noturno na noite anterior; SNF = sono noturno em dias de folga (entre 19h e 6h59min); SDF = sono diurno em dias de folga (entre $7 \mathrm{~h}$ e $18 \mathrm{~h} 59 \mathrm{~min}$ ); SNOT = sono no trabalho (entre $19 \mathrm{~h}$ e $7 \mathrm{~h}$ ); SD = sono diurno em dias de descanso (entre $7 \mathrm{~h}$ e $18 \mathrm{~h} 59 \mathrm{~min}$ ): SNCTD = sono noturno em dias de trabalho diurno (entre $19 \mathrm{~h}$ e $6 \mathrm{~h} 59 \mathrm{~min}$ ), quando houve trabalho durante o dia; SNSTD = sono noturno em dias de descanso (entre $19 \mathrm{~h}$ e $6 \mathrm{~h} 59 \mathrm{~min}$ ). Os dias de folga foram considerados a partir de $36 \mathrm{~h}$ após o término do trabalho.

existir queda significativa do alerta no decorrer das $12 \mathrm{~h}$ de trabalho diurno $(\mathrm{F}=0,02 ; \mathrm{p}=0,98)$.

\section{Discussão}

Os resultados encontrados entre os trabalhadores em turnos de $12 \mathrm{~h}$ noturnas seguidas de 36 horas de descanso apontam para uma queda significante da duração do sono após noite de trabalho. Comparados os registros das durações de episódios de sono noturno com aqueles ocorridos durante o dia, após o trabalho noturno, nota-se que o sono diurno mostrou-se fragmentado. Esta fragmentação depende de vários fatores, tais como, necessidade de cuidar de terceiros, execução de tarefas indispensáveis no âmbito familiar e social e do componente circadiano da sonolência (Akerstedt, 1998; Estryn-Behar, 1978; Gadbois, 1981; Rotenberg et al., 2001). Quando analisados os registros da duração do sono dos auxiliares e enfermeiros que trabalhavam $12 \mathrm{~h}$ diurnas, observou-se um resultado previsível, ou seja, as médias de duração dos episódios de sono noturno são maiores que as dos episódios de sono diurno. Esses trabalhadores seguem o padrão normal do ciclo vigília-sono humano, ou seja, vigília diurna e repouso noturno. Resultados similares foram encontrados por Fischer et al. (2000), quando estudados petroquímicos que também trabalhavam $12 \mathrm{~h}$ durante a noite. Naquele estudo, o esquema de trabalho em turnos era em sistema de rodízio, e havia quatro noites consecutivas de folga após trabalho noturno, o que dava aos trabalhadores possibilidades de normalizar o padrão do ciclo vigília-sono, com períodos contínuos de sono noturno, tanto durante os dias de trabalho diurno, como de folga. Essa é uma das vantagens dos turnos em sistema de rodízio, ao diminuírem a "dose de trabalho noturno" para cada trabalhador e, conseqüentemente, as alterações dos ritmos biológicos causadas e agravadas pela vigília noturna (Boggild \& Knutsson, 1999). No caso dos trabalhadores da área de enfermagem, o único dia de descanso que se seguia ao de trabalho era provavelmente insuficiente para a recuperação do débito de sono.

A respeito da percepção da qualidade dos episódios de sono para trabalhadores do turno da noite, foram observadas diferenças estatisticamente significantes entre as médias das qualidades dos episódios de sono noturno e de sono diurno. Estes últimos foram considerados de pior qualidade que os primeiros. Nos trabalhadores sujeitos a turnos diurnos, apenas foram encontradas diferenças entre a qualidade de sono diurno em dias de descanso, em comparação aos dias de trabalho. Nos dias em que não houve trabalho, as pessoas dormiram mais e melhor. Esses resultados são possivelmente decorrentes do fato de que dormem várias horas a mais em dias em que não há trabalho, pois as pessoas acordaram mais tarde nesses dias. Quando há trabalho diurno, o horário habitual de acordar foi ao redor de $5 \mathrm{~h}$ da manhã e, quando não há, foi por volta de $8 \mathrm{~h}$. 
Em relação à qualidade percebida do sono diurno após noite de trabalho: há na literatura muitos exemplos a respeito, mostrando que a qualidade do sono é pior durante o dia quando comparada à qualidade de sono noturno, após trabalho diurno. As causas são muitas, mas a principal é a falta de sincronia entre o sono diurno e os ritmos biológicos (Akerstedt \& Gillberg, 1981b; Frese \& Harwich, 1984; Lavie et al., 1989; Smith et al., 1998).

Sobre os níveis de alerta referidos, ocorreu queda significante com o passar das horas durante o turno de trabalho noturno. No decorrer do turno diurno não foram observadas diferenças significantes ao longo das horas de trabalho, e as médias dos alertas permaneceram praticamente estáveis durante as 12 horas de trabalho. Os resultados vão ao encontro dos achados de Fischer et al. (2000). No estudo realizado com petroquímicos submetidos a turnos de 12 horas diárias, o nível do alerta às $17 \mathrm{~h}$ (décima hora de trabalho) apresentou resultados semelhantes ao da sexta hora. Poder-se-ia esperar que, naquele horário $(17 \mathrm{~h})$, o alerta fosse maior, em virtude do aumento da temperatura corporal e, conseqüentemente, menor expressão de sonolência. Entretanto, sabe-se que é muito difícil dormir durante o dia em horários próximos à acrofase da temperatura (hora proibida do sono) (Lavie et al., 1989). Também é possível que o cansaço decorrente da longa jornada tenha interferido na disposição geral e, por conseqüência, no alerta registrado neste horário, que seria maior se a pessoa não estivesse muito cansada. Acrescente-se a isto o fato de não ter sido observado um importante débito acumulado de sono nos trabalhadores dos turnos diurnos, o que provavelmente diminuiu a vontade de dormir nesse horário de final de tarde (Akerstedt \& Gillberg, 1981a, 1986). A queda no alerta à noite é esperada porque neste período ocorre o menor valor da expressão do componente circadiano da regulação do alerta, aumento da fadiga e débito de sono, gerando maior sonolência (Akerstedt, 1996). Segundo Akerstedt (1998), esse fato também pode ocorrer porque, ao final do trabalho noturno, um trabalhador pode alcançar um tempo de vigília de 20 a $22 \mathrm{~h}$, contadas a partir do último episódio de sono, em comparação com até 13h de vigília para trabalhadores diurnos.

Existem controvérsias em relação à duração da jornada de trabalho e suas repercussões no alerta (Duchon \& Smith, 1993; Rosa et al., 1989; Smith et al., 1998). Duchon \& Rosa em suas publicações apresentam resultados negativos tanto no alerta, quanto no desempenho, em pessoas trabalhando $12 \mathrm{~h}$ diárias. Não é unânime

\begin{tabular}{|c|c|c|c|}
\hline \multicolumn{4}{|c|}{$\begin{array}{l}\text { Médias e desvios-padrão da percepção do alerta. Turnos diurno e noturno } \\
\text { de } 12 \mathrm{~h} \text { por } 36 \mathrm{~h} \text { de descanso, segundo período de trabalho. Hospital das Clínicas, } \\
\text { São Paulo, } 1999 .\end{array}$} \\
\hline Horário de coleta & $\mathbf{n}$ & Média & DP \\
\hline \multicolumn{4}{|l|}{ Trabalho noturno } \\
\hline 21:00 & 86 & 8,58 & 1,57 \\
\hline $01: 00$ & 84 & 6,54 & 2,56 \\
\hline 05:00 & 74 & 5,04 & 3,30 \\
\hline \multicolumn{4}{|l|}{ Trabalho diurno } \\
\hline 09:00 & 63 & 6,83 & 2,75 \\
\hline $13: 00$ & 60 & 6,67 & 2,50 \\
\hline $17: 00$ & 59 & 6,77 & 2,67 \\
\hline
\end{tabular}

$\mathrm{n}=$ número de julgamentos para o Estado de Alerta Subjetivo.

Figura 1

Desvios padrão das percepções de alerta registradas ao longo dos turnos de 12 horas. Hospital da Clínicas, São Paulo, 1999.

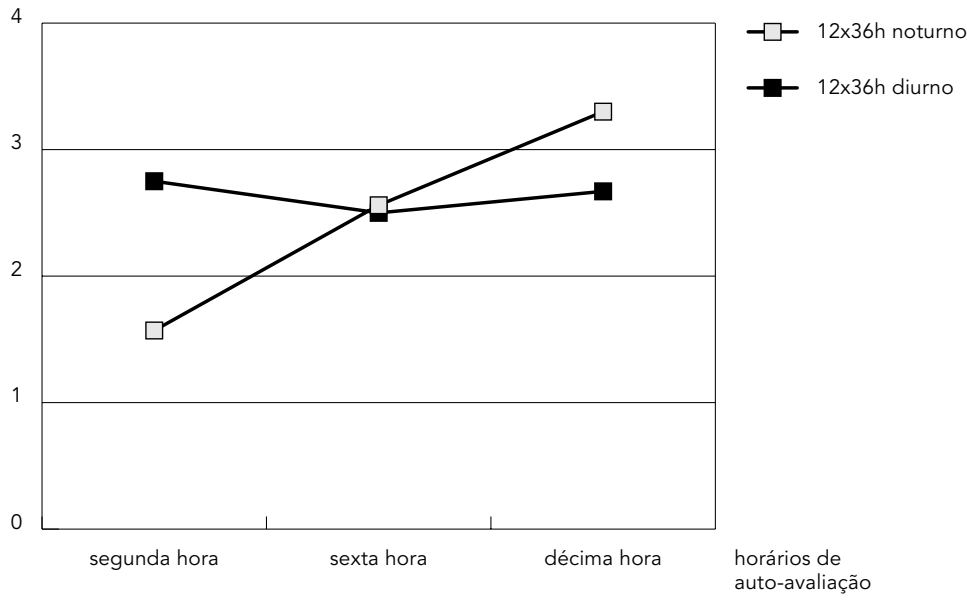

entre os autores que $12 \mathrm{~h}$ representem um tempo muito prolongado, pois em revisão recente de Smith et al. (1998) não foram encontrados impactos negativos sobre a saúde. Entretanto, os autores mencionados acima não descartam que há necessidade de serem avaliados os impactos das exigências do trabalho, especialmente físicos, e outros efeitos que possam exacerbar os efeitos das cargas de trabalho no aparecimento de doenças, levando a um envelhe- 
cimento funcional precoce. É necessária uma cuidadosa avaliação sobre a exposição ocupacional a diversos agentes estressores durante turnos de trabalho prolongados, antes de ser decidido se um particular esquema de turnos de trabalho prolongado tem ou não efeitos adversos sobre a saúde e o desempenho dos trabalhadores. Em relação às diferenças individuais nas respostas sobre o alerta, pode ser observado que as médias e desvio-padrão das pessoas que trabalharam durante o dia não se modificaram ao longo da jornada com a mesma intensidade das respostas das pessoas que trabalhavam à noite. Ou seja, ocorreu maior variabilidade individual na percepção do alerta à medida que se tornava mais difícil permanecer acordado. Isso significa que as diferenças individuais tornam-se mais importantes quando a situação de trabalho apresenta maiores dificuldades, logo é possível supor que, ao longo do tempo, a população que permanece trabalhando é a "sobrevivente", ocorrendo uma auto-seleção importante entre os trabalhadores notur-

\section{Agradecimentos}

Aos participantes do estudo; ao Conselho Nacional de Desenvolvimento Científico e Tecnológico (Processo 52.1981/96-4), pelo apoio financeiro; à direção do Instituto Central do Hospital das Clínicas da Faculdade de Medicina da Universidade de São Paulo, que tornou possível realização deste trabalho. nos. Daí a importância de manter um processo contínuo de monitoramento da saúde na população trabalhadora.

Embora os resultados observados neste estudo tenham sido obtidos avaliando um reduzido número de profissionais, o que traz limitações à generalização dos resultados, é provável que essa realidade seja encontrada entre outros trabalhadores de hospitais que estejam submetidos a condições de trabalho e de vida similares às daqueles desta amostra estudada. Tendo em vista que os níveis percebidos de alerta durante a noite tornam-se piores à medida que aumenta o número de horas de trabalho, tanto os trabalhadores, quanto os pacientes que estão aos seus cuidados, podem ser prejudicados seriamente. Isso levanta a necessidade de que seja revista a tradição dos turnos de $12 \mathrm{~h}$ de trabalho por $36 \mathrm{~h}$ de descanso no setor de enfermagem, particularmente em postos de trabalho onde há importantes demandas físicas e cognitivas, além de exposição a estressores ocupacionais.

\section{Referências}

AKERSTEDT, T., 1996. Wide Awake at Odd Hours Shiftwork, Timezones and Burning the Midnight Oil. Stockholm: Swedish Council for Work, Life and Research.

AKERSTEDT, T., 1998. Is there an optimal sleep-wake pattern in shift work? Scandinavian Journal of Work, Environment \& Health, 24:18-27.

AKERSTEDT, T. \& GILLBERG, M., 1981a. Sleep disturbances and shiftwork. In: Night and Shift Work. Biological and Social Aspects (A. Reinberg, N. Vieux \& P. Andlauer, ed.), pp. 127-137, Oxford: Pergamon Press.

AKERSTEDT, T. \& GILLBERG, M., 1981b. The circadian variations of experimentally displaced sleep. Sleep, 4:159-169.

AKERSTEDT, T. \& GILLBERG, M., 1986. A dose response study of sleep loss and spontaneous sleep termination. Psychophysiology, 23:293-297.

BOGGILD, H. \& KNUTSSON, A., 1999. Shift work, risk factors and cardiovascular disease. Scandinavian Journal of Work, Environment and Health, 25:85-99. 
DINGES, D. F; PACK, F; WILLIAMS, K.; GILLEN, K. H. \& POWELL, J. W., 1997. Cumulative sleepiness, mood disturbance and psychomotor vigilance performance decrements during a week of sleep restricted to 4-5 hour per night. Sleep, 20:266-277.

DUCHON, J. C. \& SMITH, T.J., 1993. Extended workdays and safety. International Journal of Industrial Ergonomics, 11:37-49.

ESCRIBA, V.; PEREZ-HOYOS, S. \& BOLUMAR, F., 1992. Shiftwork: Its impact on the length and quality of sleep among nurses of the Valencian region in Spain. International Archives of Occupational and Environmental Health, 64:125-129.

ESTRYN-BEHAR, M.; GADBOIS, C. \& VAICHERE, E., 1978. Effets du travail de nuit en équipes fixes sur une population féminine. Résultats d'une enquête dans le secteur hospitalier. Archives des Maladies Profissionelles de Médecine du Travail et de Sécurité Sociale, 39:531-535.

FISCHER, F. M.; BELLUSCI, S. M.; TEIXEIRA, L. R.; BORGES, F. N. S.; FERREIRA, R. M.; GONÇALVES, M. B. L.; MARTINS, S. E. \& CHRISTOFFOLETE, M. A., 2002. Unveiling factors that contribute to functional aging among health care shiftworkers in São Paulo, Brazil. Experimental Aging Research, 28:73-86.

FISCHER, F. M.; HOFMEISTER, V. A.; SCATENA, J. C. \& BRUNI, A. C., 1987. Reorganização do trabalho em turnos na companhia do metropolitano de São Paulo. In: Congresso da Associação Nacional de Medicina do Trabalho, Anais, v. 2, pp. 677-692, Florianópolis: Associação Nacional de Medicina do Trabalho.

FISCHER, F. M.; MORENO, C. R. C.; BORGES, F. N. S. \& LOUZADA, F. M., 2000. Alertness and sleep after 12-hour shifts: Differences between day and night in work. In: Shiftwork the $21^{\text {st }}$ Century (S. Hornberger, P. Knauth, G. Costa \& S. Folkard, ed.), Arbeitswissenschaft in der betrieblichen Praxis, v. 17, pp. 43-48, Frankfurt: Peter Lang.

FONSECA, J. S. \& MARTINS, G. A., 1996. Curso de Estatística. São Paulo: Editora Atlas.

FRESE, M. \& HARWICH, C., 1984. Shiftwork and the length and quality of sleep. Journal of Occupational Medicine, 26:561-566.

GADBOIS, C., 1981. Aides soignantes et infirmières de nuit: Conditions de travail et vie quotidienne. Paris: ANACT.
GOLD, D., 1992. Rotating shift work, sleep, and accidents related to sleepiness in hospital nurses. American Journal of Public Health, 82:1011-1014.

KNAUTH, P.; PLETT, R.; CONDON, R.; DEVOL, D.; FLETCHER, N.; EICKHOFF, S. \& OTTMANN, W., 1983. Sleep duration and subjective ratings of sleep quality determined by a permanent watch keeping system. In: Night and Shiftwork: Longterm Effects and their Prevention (M. Haider, M. Koller \& R. Cervinka, ed.), pp. 293-310, 1st Ed. Frankfurt: Peter Lang.

LAVIE, P.; CHILLAG, N.; EPSTEIN, R.; TZISCHINSKY, O.; GIVON, R.; FUCHS, S. \& SHAHAL, B., 1989. Sleep disturbances in shift workers: A marker for maladaptation syndrome. Work and Stress, 3:33-40.

MENEZES, G. M. S. M., 1996. Trabalho Noturno e Saúde: Um Estudo com Profissionais de Enfermagem de um Hospital Público de Salvador, Bahia. Dissertação de Mestrado, Salvador: Instituto de Saúde Coletiva, Universidade Federal da Bahia.

NIEDHAMMER, M. S.; LERT, F. \& MARNE, M. J., 1994. Effects of shift work on sleep among French nurses. Journal of Occupational Medicine, 36:667-674.

ROSA, R. R.; COLLIGAN, M. J. \& LEWIS, P., 1989. Extended workdays: Effects of 8-hour and 12-hour rotating shift schedules on performance, subjective alertness, sleep patterns, and psychosocial variables. Work and Stress, 3:21-32.

ROTENBERG, L.; PORTELA, L. F.; MARCONDES, W. B.; MORENO, C. R. C. \& NASCIMENTO, C. P., 2001. Gênero e trabalho noturno: Sono, cotidiano e vivências de quem troca a noite pelo dia. Cadernos de Saúde Pública, 17:639-649.

SIEGEL, S., 1975. Estatística Não-Paramétrica (Para Ciências do Comportamento). São Paulo: McGraw-Hill.

SMITH, L.; FOLKARD, S.; TUCKER, P. \& MacDONALD, I., 1998. Work shift duration: A review comparing eight hour and 12 hour shift systems. Occupational and Environment Medicine, 55:217-229.

TEPAS, D. I., 1996. Survey of Health Care Professionals. Storrs: Connecticut University.

TUOMI, K.; ILMARINEN, J.; JAHKOLA, A.; KATAJARINNE, L. \& TULKKI, A., 1997. Work Ability Index. Helsinki: Institute of Occupational Health.

Recebido em 14 de agosto de 2001

Versão final reapresentada em 21 de novembro de 2001 Aprovado em 27 de fevereiro de 2002 